Historic, Archive Document

Do not assume content reflects current scientific knowledge, policies, or practices. 



\title{
S E E D S
}

\author{
OF
}

\section{NATIVE TREES, SHRUBS}

\section{AND PLANTS}

Crop of 1931

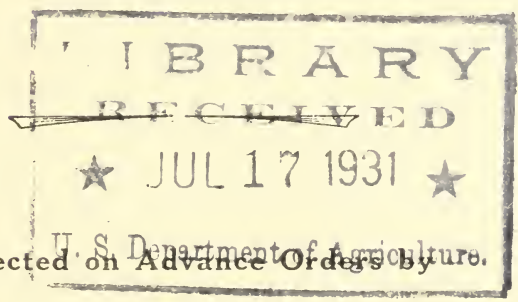

J. M. BASSETT

21 N. Packard Street

HAMMONTON, N. J., U. S. A. 


\section{VARIETIES}

per lb.

Amelanchier Canadensis............................\$3.00

Amorpha Fruticosa.................................. $\quad .75$

Ampelopsis Quinquefolia.......................... 1.50

Andromeda Mariana, pods........................ 1.25

“ $\quad$ Ligustrina, pods..................... 1.25

“ Racemosa, pods..................... 1.25

“ Calyculata, pods.................. 2.00

Aquilegia Canadensis,....oz. $\$ 1.00$

Aronia Arbutifolia, berries..................... . .50

Asclepias Tuberosa..........oz. $\$ 2.00$

Azalea Viscosa, pods................................. 4.00

Betula Populifolia, $10 \mathrm{lbs}$. at $85 \mathrm{c} . . . . . . . . . . . . .1 .00$

Cephalanthus Occidentalis........................ $\quad .75$

Clethra Alnifolia...................................... . .75

Comptonia Asplenifolia.............................. 3.00

Cornus Amomum........................................ 1.00

Crategus Crusgalli...................................... 1.00

، Parvifolia ..................................... 1.00

Cupressus Thyoides, pods........................... 4.00

Diospyrus Virginiana................................ $\quad .50$

Gaultheria Procumbens............................ 4.00

Gaylussacia Dumosa................................... 1.50

“ Frondosa ............................. 1.25

". Resinosa ............................... 1.25

Hibiscus Mocheutos..................................... 4. 4.00

Ilex Glabra............................................... 2.00

“ Opaca ................................................... $\quad .75$

“ Verticillata .............................................. . .75

Itea Virginica.......................................... 2.00

Juniperus Virginiana................................. 1.50

Kalmia Angustifolia, pods........................ 1.50

“ Latifolia, pods................................ 1.50

clean ........................... 8.00

All quotations subject to crop failure, forest fires or other conditions beyond our control. 
Leiophyllum Buxifolium.......................... 4.00

Lupinus Perennis........oz. 40 c................... 5.00

Magnolia Glauca, berries........................ 2.50

“ Fresh Cones....per bu. $\$ 8.00$

Myrica Cerifera........................................ .75

Nyssa Sylvatica........................................ 1.50

Pinus Echinata..1 lb. $\$ 10 . .10 \mathrm{lbs}$, or over 8.00

“ Rigida......\$6.00....10 lbs. or over 5.00

“. Virginiana .................................... 6.00

Platanus Occidentalis.............................. $\quad .50$

Prunus Maritima.................................... 2... 2.00

Quercus Illicifolia.................................... $\quad .20$

“ $\quad$ Prinoides .................................... .25

“ Prinos .......................................... .15

Rhus Copalina.......................................... $\quad .50$

" Glabra .............................................. . .75

Rosa Carolina............................................. . .75

“ Lucida ........................................... 1.00

Sassafras Officinalis............................... 1.50

Smilax Rotundifolia................................ 1.00

، Walterii ..................................... 3.00

Tephrosia Virginica............oz. 25c........... 3.00

Vaccinium Corymbosum........................... 3.50

“ Vascellans ............................. 2.50

Vilurnum Cassinoides................................ 1.00

“ Dentatum .................................. $\quad .75$

، Nudum ....................................... $\quad .75$

\section{Order Early}

Varieties that mature early should be ordered as far in advance as possible to avoid any disappointment.

Prices subject to change without notice. 


\section{ORDER EARLY-}

As I collect seeds on orders only, I seldom have much surplus to fill late orders, but customers are assured of fresh crop seeds.

All orders are subject to conditions of the season's growth-crop failure, forest fires, etc.

Terms Cash with order, or satisfactory references.

I personally attend to the collection of all native seed orders and handle only such as are mature. 